developed methicillin-sensitive $S$ aureus (MSSA) infection. They surveyed 1,417 ICUs in 17 Western European countries. There were 10,038 patients in ICUs who were part of the European Prevalence of Infection in Intensive Care (EPIC) study. Prevalence of MRSA and MSSA ICU-acquired infections, risk factors, and mortality were determined.

On the study day, $21 \%$ of patients had ICU-acquired infections. The most commonly reported pathogen was $S$ aureus (30\%). Overall, $60 \%$ of $S$ aureus strains were resistant to methicillin (with a wide intercountry variation). The most commonly reported MRSA infections were pneumonia and lower respiratory tract infections. The most important risk factor for MRSA was the length of stay in the ICU. MRSA infection reduced the chance of survival, particularly when it was found in lower respiratory tract infections. The risk of mortality was three times higher in patients with MRSA than in those with MSSA. The authors concluded that patients in ICU are at high risk of becoming infected with MRSA; the longer they stay, the higher the risk. Patients with MRSA infections are less likely to survive than those with MSSA.

FROM: Ibelings MM, Bruining HA. Methicillin-resistant Staphylococcus aureus: acquisition and risk of death in patients in the intensive care unit. Eur J Surg 1998;164:411-418.

\section{Impact of Clindamycin Restriction on Clostridium difficile}

Widespread antibiotic use has been associated with increases in both bacterial resistance and nosocomial infection. Climo and coinvestigators from Hunter Holmes McGuire Veterans' Hospital characterized the impact of hospitalwide clindamycin restriction on the incidence of Clostridium difficile-associated diarrhea $(\mathrm{CDAD})$ and on antimicrobial prescribing practices.

An outbreak of CDAD was found to be caused by a clonal isolate of clindamycin-resistant $C$ difficile and was associated with increased use of clindamycin. Hospitalwide requirement of approval by an infectious disease consultant of clindamycin use led to an overall reduction in clindamycin use, a sustained reduction in the mean number of cases of CDAD (11.5 cases/mo vs 3.33 cases $/ \mathrm{mo} ; P<.001)$, and an increase in clindamycin susceptibility among $C$ difficile isolates ( $9 \%$ vs $61 \% ; P<.001)$. A parallel increase was noted in the use of, and costs associated with, other antibiotics with antianaerobic activity, including cefotetan, ticarcillin-clavulanate, and imipenem-cilastin. The hospital realized overall cost savings as a result of the decreased incidence of CDAD.

Hospital formulary restriction of clindamycin was found to be an effective way to decrease $\mathrm{CDAD}$. It also can lead to a return in clindamycin susceptibility among isolates and can effect cost savings to the hospital.

FROM: Climo MW, Israel DS, Wong ES, Williams D, Coudron P, Markowitz SM. Hospital-wide restriction of clindamycin: effect on the incidence of Clostridium difficile-associated diarrhea and cost. Ann Intern Med 1998;128:989-995.

\section{Chart Reminders for Pneumococcal Vaccination}

Researchers from the University of Oklahoma Health Science Center reported the results of a pharmacy-based program to increase pneumococcal vaccination rates using simple chart reminders. On a daily basis, inpatient records on general medicine and cardiology services at an academic medical center were reviewed to determine which patients were eligible to receive pneumococcal vaccine. Eligible inpatients were interviewed, and the percentage of nonvaccinated inpatients given vaccine during hospitalization was determined. During an intervention period, reminders were placed on charts requesting a vaccine when indicated.

Of 447 inpatients, 224 (50.1\%) had one or more indications for receiving pneumococcal vaccine but only $64(28.6 \%)$ had been vaccinated previously. Of 224 vaccine-eligible patients, 158 (70.5\%) had a prior hospitalization within the previous 5 years. Previous hospitalization was not significantly associated with receiving (48 [30.4\%] of 158) or not receiving (16 [24.2\%] of $66 ; P=.35$ ) vaccine prior to admission. During the observational period, 0 of 80 vaccine-eligible, nonvaccinated inpatients were vaccinated before discharge. In comparison, 23 $(29 \%)$ of 80 inpatients were vaccinated after a chart reminder $(P<.001)$. During the intervention period, vaccination rates were $10-$ fold higher on general medicine services than on cardiology services.

The authors concluded that a hospital-based pharmacy vaccination program that relied on simple chart reminders was significantly associated with increased vaccination rates among inpatients at risk for invasive pneumococcal disease.

FROM: Vondracek TG, Pham TP, Huycke MM. A hospitalbased pharmacy intervention program for pneumococcal vaccination. Arch Intern Med 1998;158:1543-1547.

\section{Group B Streptococcal Necrotizing Fasciitis}

Necrotizing fasciitis, a severe and uncommon infection involving the subcutaneous tissues, usually is caused by group A streptococci; group B streptococci (Streptococcus agalactiae) have been reported to cause necrotizing fasciitis in only four instances (two involving neonates) over the past 4 decades. Researchers from Sir Mortimer B. Davis-Jewish General Hospital and McGill University, Montreal, Quebec, Canada, reported three cases of group B streptococcal necrotizing fasciitis in adults in southern Ontario and Quebec within a 10-month period. All three patients had serious underlying illness, and all required surgical debridement in addition to antibiotic therapy. One of the cases fulfilled the criteria for streptococcal toxic shock-like syndrome. Group B streptococcus has been recognized as a frequent cause of serious disease in adults. It has become evident over the past decade that invasive streptococcal infections are on the increase. The authors suggest that group B streptococcus recently has acquired an increased ability to cause necrotizing fasciitis and suggest that this may represent the emergence of a new clinical syndrome in adults.

FROM: Gardam MA, Low DE, Saginur R, Miller MA. Group B streptococcal necrotizing fasciitis and streptococcal toxic shocklike syndrome in adults. Arch Intern Med 1998;158:1704-1708.

\section{Biofilms in Hemodialysis Tubing}

Man and coinvestigators conducted a study of biofilms associated with hemodialysis machines. Biofilms consist of microorganisms immobilized at a substratum surface embedded in an organic polymer matrix of bacterial origin. Tubing drawn from the fluid pathways within dialysis machines of various models were investigated for biofilm.

Scanning electron microscopy (SEM) performed on approximately $2-\mathrm{cm}^{2}$ samples of the tubing inner surfaces revealed that the inner surfaces of the tubing were covered with biofilms consisting of numerous deposits and glycocalix at different stages of formation, with components containing bacteria and algae. Evaluations of biomass were performed from tubing sections of various lengths and inner diameters put in tubes containing water for injection and 